\title{
Barreras sonoras: Posible solución para el intercambio vial Av. Venezuela y Universitaria. Estudio preliminar.
}

\author{
Sound barriers: Possible solution for vial sharing Venezuela and \\ University Avenue, preliminary study
}

YARIN ACHACHAGUA, Anwar Julio'; LLOSA DEMARTINI, Melchor?; HERENCIA CALAMPA, Nicolás; GOMEZ BARRIA, Javier

\section{RESUMEN}

Se relata una posible solución al incremento de ruido esperado por el flujo automovilístico producido por las obras del intercambio vial de las Avenidas Universitaria y Venezuela y que no ha sido considerada en la propuesta inicial que viene siendo ejecutada por la Municipalidad de Lima Metropolitana. Las barreras sonoras son muy comunes en los países desarrollados, pero vemos que dicha tecnología no ha sido introducida en nuestro medio y esta obra al estar colindante a la principal y más importante universidad peruana merece esta mejora para no afectar los procesos de enseñanza aprendizaje (PEA).

Palabras clave: Barreras sonoras, rayos acústicos, seguridad ambiental.

\begin{abstract}
A possible solution to the increase of noise waited for by the automobile flow produced by works of the road interchange of the Avenues Universitaria and Venezuela and who have not been considered in the initial proposal that comes being executed by the Metropolitan Municipality of Lima is related. The sonorous barriers are very common in the developed countries, but we see that this technology has not been introduced in our means and this work when adjacent being to the main and more important Peruvian university deserves this improvement not to affect the education processes learning (EPL).
\end{abstract}

Key words: Noise Barriers, Ray Acoustics, Environmental safety. 


\section{INTRODUCCIÓN}

Las barreras sonoras proporcionan protección a las poblaciones cercanas, pero para que estas funcionen correctamente es esencial que los diseñadores entiendan los principios básicos de la teoría acústica de la barrera. El sonido propaga de una fuente como una serie de ondas de presión que fluctúan rápidamente las cuales se expanden esféricamente mientras se mueven lejos de la fuente (Figura 1a). Estas ondas de presión crean la sensación de ruido cuando alcanzan el oído del oyente. Aunque el sonido viaja como onda es frecuentemente conveniente modelar la propagación del sonido como líneas rectas o rayos, que alcanzan el oyente o el receptor directamente o indirectamente después de ser reflejado o difractado por las superficies de intervención (Figura 1b). El método del trazado de rayos (ray-tracing) se utilizará para esta investigación.
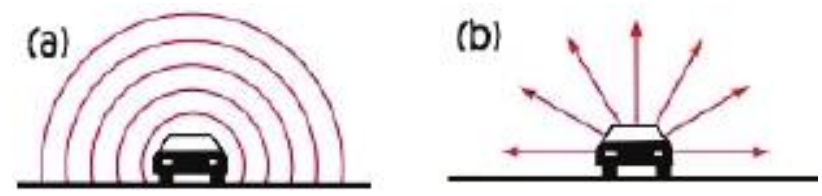

Figura 1. Propagación del sonido. (a) Propagación esférica del sonido. (b) Modelo de rayos.

\section{TEORIA}

Para un camino descubierto, la trayectoria más importante de la transmisión sonora es el rayo que viaja

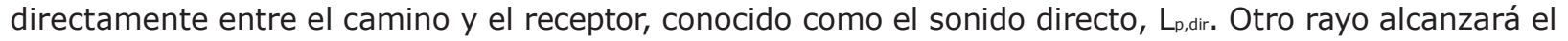
receptor es el rayo que pega la tierra y se refleja hacia el receptor, $L_{p}$, grd (Figura 2). Hay un grado de interferencia destructiva entre estos dos rayos que da lugar a la mayor atenuación del $L_{p}$, dir que es esperado por extensión geométrica solamente.

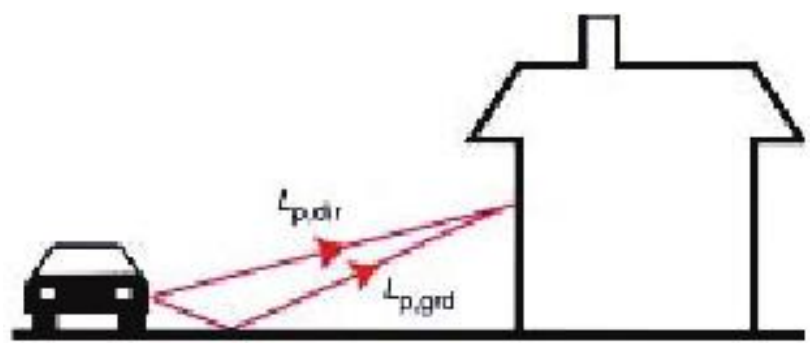

Figura 2. Trayectoria de la transmisión sonora no obstruida.

El mecanismo exacto de esta atenuación no está completamente entendido, solamente es mejor cuando la propagación acústica es por ejemplo sobre el césped, y donde está el $L_{p}$, dir particularmente cerca de la tierra. Esta atenuación de tierra es dependiente de la frecuencia y Hutchins [1, 2] ha demostrado que la interferencia destructiva ocurre predominante en una gama de frecuencia centrada en 500 hertz (Figura 3).

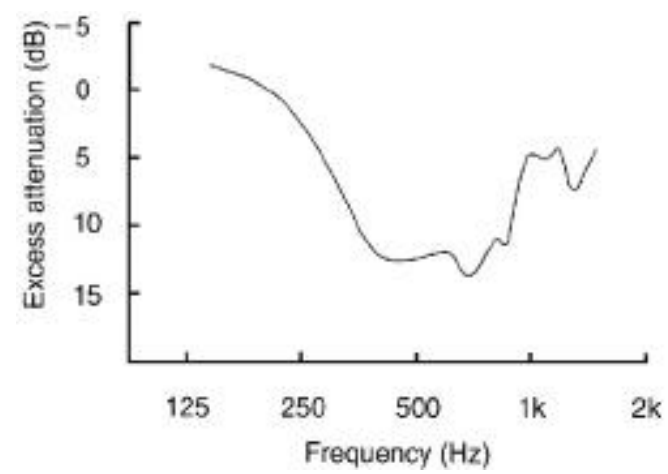

Figura 3. Atenuación excesiva por propagación sobre una base suave. 
La introducción de una barrera puede reducir bastante bien la fuerza del rayo directo, aunque para la

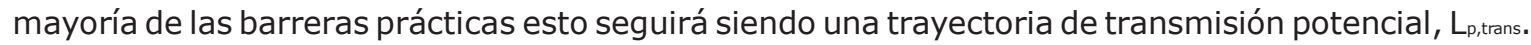
El rayo importante es ahora que está difractado hacia abajo del borde superior de la barrera, $L_{p, \text { diff }}$ (Figura 4). La presencia de la barrera también elimina el $\mathrm{L}_{\mathrm{p}}$ grd como trayectoria significativa de la transmisión sonora.

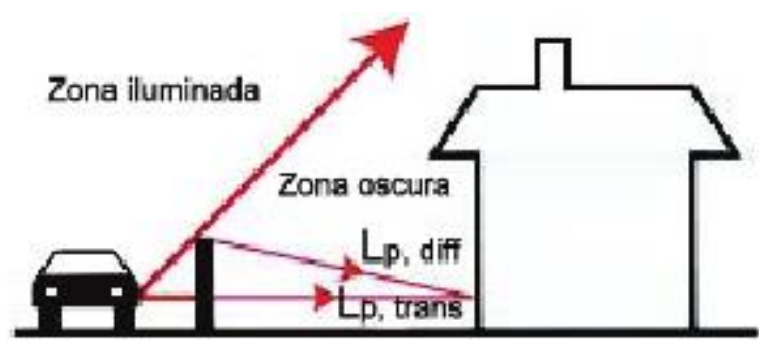

Figura 4. Forma del apantallamiento de la transmisión del sonido por una barrera sonora.

Este trabajo se ha realizado, usando técnicas del ray-tracing, para establecer el funcionamiento acústico de una barrera, a saber la diferencia entre el $L_{p}$, dir $y$ el $L_{p}$, diff. El trabajo más influyente es probablemente el de Maekawa [3] y éste es la base la mayor parte de de los métodos prácticos para calcular funcionamiento de la barrera. Otros desarrollaron la teoría básica de la barrera y correlacionaron los resultados como Rathe [4], la teoría desarrollada calcula el funcionamiento acústico de una pantalla vertical adentro términos del número de Fresnel $\mathrm{N}$, que se define como:

$$
N=2 \frac{\delta}{\lambda}
$$

donde $\square$ es la diferencia de la longitud de trayectoria (longitud de trayectoria difractada menos la longitud de trayectoria directa) y el $\boldsymbol{\lambda}$ es la longitud de onda del sonido en aire. En la zona de la sombra, el área donde la barrera rompe la visión entre la fuente y el receptor, $\boldsymbol{\delta}$ se define como positivo, y para los rayos que propagan sobre el borde de difracción de la barrera, en la zona iluminada, el $\boldsymbol{\delta}$ es negativo. Kurze y Anderson [5] dieron la ecuación siguiente para la pérdida de inserción (insertion loss, IL) de una barrera:

$$
\begin{aligned}
& I L=5+20 \log \frac{\sqrt{2 \pi N}}{\tanh \sqrt{2 \pi N}}(d B) \quad \text { para }-0.2<\mathrm{N}<12.5 \\
& =24(\mathrm{~dB}) \text { para } \mathrm{N}>12.5
\end{aligned}
$$

La fórmula anterior se aplica solamente vehículos en su punto más cercano al receptor y expresiones más complejas están disponibles para describir el funcionamiento de una barrera para un flujo denso de tráfico. Afortunadamente para los diseñadores de barreras, usualmente no necesitan utilizarlos mientras que los métodos aprobados del cálculo para el ruido del tráfico proporcionan los resultados en forma gráfica o tabular, y un programa de computadora puede ejecutar estos métodos dados. La ecuación (2) puede ser usada cuando una barrera apenas rompe la visión entre la fuente de ruido y el receptor y hay una atenuación de $5 \mathrm{~dB}$ de ruido, y puede haber una cierta reducción del ruido para los receptores en la zona iluminada. La atenuación de la barrera usada en el método de cálculo del ruido de trafico en el Reino Unido [6] se demuestra en la figura 5. Esto da un límite teórico para la atenuación de la barrera de cerca de 20 $\mathrm{dB}(\mathrm{A})$ en la zona de la sombra; sin embargo, los valores requeridos de $\boldsymbol{\delta}$ pueden ser observados raramente y, en la práctica, un límite realista es de cerca de $15 \mathrm{~dB}(\mathrm{~A})$. El gráfico también muestra que en la zona iluminada la atenuación de la barrera tiende rápidamente a cero en $\boldsymbol{\delta} \leqslant-0.6$ y por lo tanto hay poca ventaja práctica de la investigación que se ganará en esta región.

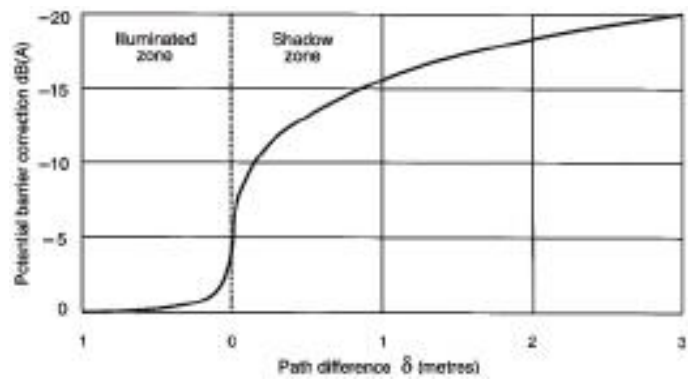

Figura 5. Corrección de la barrera potencial en función de la diferencia de trayectoria. 


\section{ANALISIS CUANTITATIVO}

Haremos nuestros cálculos considerando la colocación de una barrera voladiza, ya que esta es más estética y evita la alteración del paisaje además de esta diseñada para evitar la propagación en lugares altos, que es exactamente lo que queremos evitar (ver figura 6).

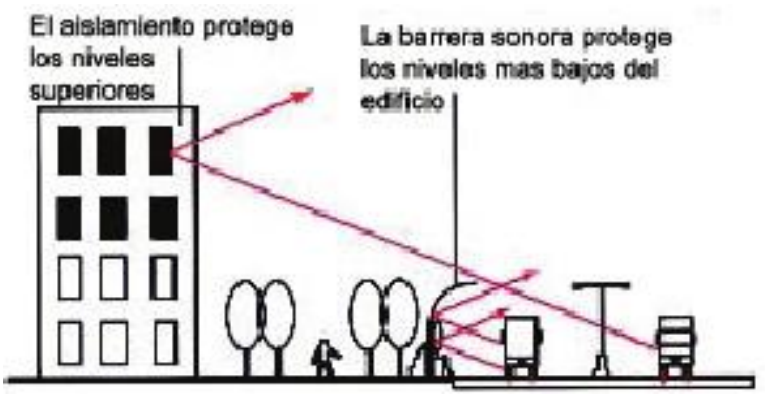

Figura 6. Forma de protección de una barrera voladiza.

Podemos apreciar que en el lado de la Av. Venezuela, tenemos los frontis de la Facultad de Ing. Industrial, Ciencias Físicas y Ciencias Matemáticas, los que son los más perjudicados por el incremento en los niveles de ruido. Si tomamos como ejemplo el frontis de Ing. Industrial este posee una altura medida de 7.9 metros, considerando la envergadura de la Av. Venezuela (ancho variable, pero de $20 \mathrm{~m}$ en esta zona) y la nueva separación de la avenida auxiliar, por geometría simple, se necesitaría una barrera de una altura de 3 metros.

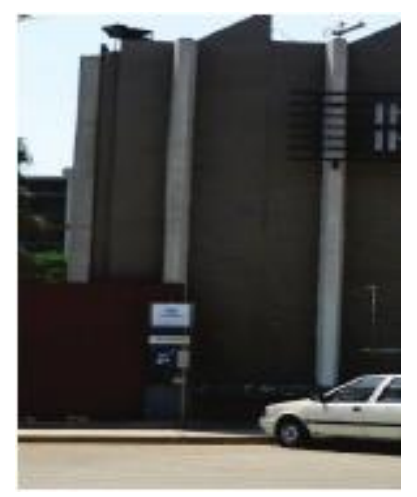

Figura 7. Frontis de la Facultad de Ing. Industrial

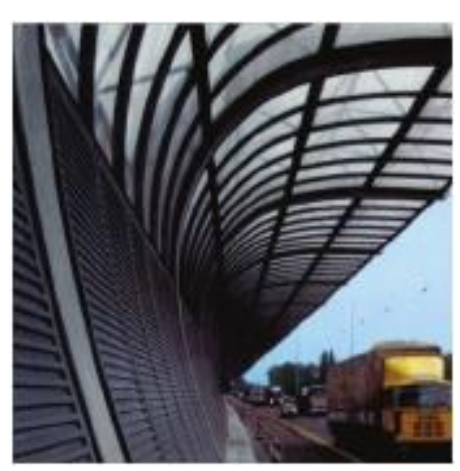

Figura 8. Detalle de la barrera voladiza.

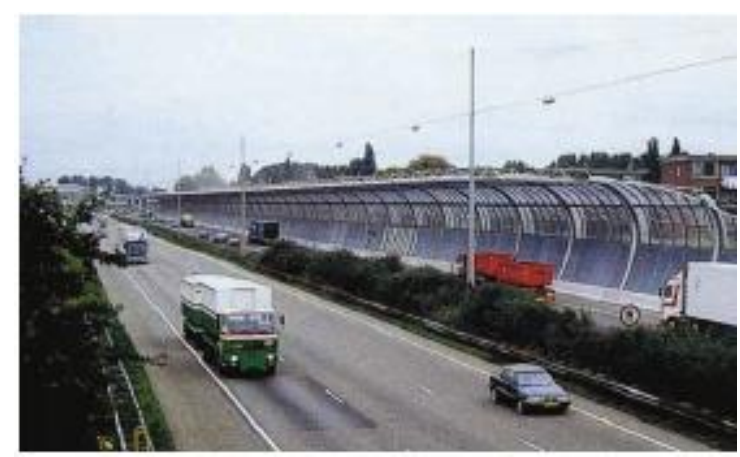

Figura 9. Vista de la barrera voladiza, no altera el paisaje y crea una sensación de modernidad. 


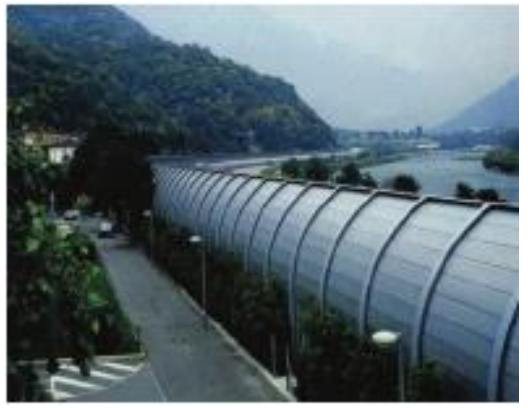

Figura 10. Detalle del lado posterior de la barrera voladiza, la cual seria vista desde el interior de la ciudad Universitaria.

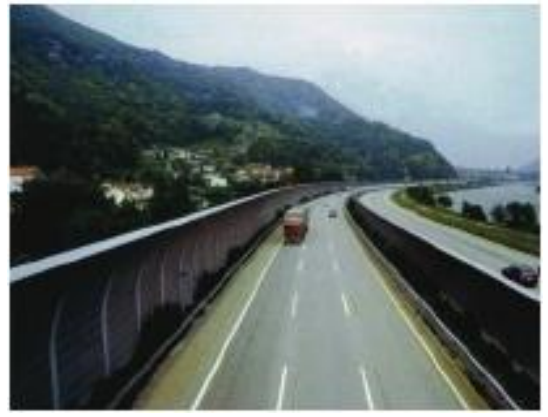

Figura 11. Vista en los dos carriles que seria factible colocar en la avenida Venezuela.

\section{CONCLUSIÓN}

El aislamiento sonoro proporcionado por una barrera es dependiente de muchos factores por ejemplo masa superficial, tiesura (rigidez acústica), factores de pérdida y el ángulo de la incidencia del sonido. El más significativo de éstos es la masa superficial de la barrera. Sin embargo estos primeros cálculos nos muestra la factibilidad de usar la barrera sonora como una posible solución al incremento de ruido en el perímetro de la UNMSM. Los cálculos posteriores son motivo de una posterior investigación en la cual se comprometen los autores y tema de tesis de grado de maestro del autor principal.

\section{REFERENCIAS BIBLIOGRÁFICAS}

1. Hutchins, D. A., Jones, H W. and Russel, L. T. "Model studies of barrier performance in the presence of ground surfaces, Part 1 - Thin perfectly reflecting barriers". Journal of the Acoustical Society of America, 75(6), 1807-16. (1984).

2. Hutchins, D. A., Jones, H W. and Russel, L. T. "Model studies of barrier performance in the presence of ground surfaces, Part 2 - Different shapes". Journal of the Acoustical Society of America, 75(6), 1817-26. (1984).
3. Maekawa, Z. "Noise reduction by screens", Journal of Applied Acoustic 1, 157-73. (1968)

4. Rathe, E. J. "Note on two common problems of sound attenuation", Journal of Sound and Vibration, 10(3), 472-9. (1969).

5. Kurze, U. J. and Anderson, G. S. "Sound attenuation by barriers", Applied Acoustic, 4, 35-53. (1971).

6. Departament of Transport and Welsh Office. Calculation of Road Traffic Noise, HMSO, London. (1988). 